\title{
STUDY OF FLOW DYNAMICS IN THE MODEL OF CARGO TRANSPORTATION ORGANIZATION BETWEEN NODE STATIONS
}

\author{
Nerses K. Khachatryan \\ State Academic University for the Humanities \\ Maronovskiy pereulok, 26 \\ 119049 - Moscow, RUSSIA
}

\begin{abstract}
This article presents a model of the organization of railway cargo transportation on a section of the railway network between two node stations. All stations have the same characteristics, such as the number of roads on them, the efficiency of their use, as well as the capacity. The latter characteristic is defined as the maximum amount of transported cargo per unit of time, depending on the load of the stations, i.e. on the number of involved roads. Using a a system of differential equations with discontinuous right parts, the intensity of cargo flow is set, which depends on the intensity of the supply of goods to the initial nodal station and the rules for moving goods from one station to another. A numerical realization of this system is carried out, with the help of which the dynamics of the load of stations and the flow arising in the system of cargo transportation are investigated.
\end{abstract}

AMS Subject Classification: 34A36

Key Words: organization of railway cargo transportation; flow intensity; stationary flow; differential equations; numerical realization

\section{Introduction}

Transport plays an important role in the country's economy. It ensures the development, communication and coordination of the work of all sectors of the

Received: August 26, 2020

(C) 2020 Academic Publications 
economy, and also the connection between the regions of the country. The development of transport is the most important condition for the modernization of the economy. It is impossible without optimal planning of networks, improving the organization of traffic and a number of other problems, the solution of which is impossible without mathematical modeling of traffic flows. Two major areas can be distinguished here: modeling the loading of urban transport networks and modeling of traffic dynamics (see [1]). The first direction is represented by models of correspondence calculation such as the gravitational model (see [2]), the entropy model (see [3]), the models of a family of competing destinations (see [4], [5]), as well as models of flow distribution, including various options equilibrium distribution and model of optimal strategies (see [6], [7], [8], [9]). The second direction is represented by the main classes of dynamic models macroscopic and microscopic. Macroscopic model (see [10], [11], [12]) describe the movement of vehicles in average terms, such like density, average speed, flow, etc. With this approach, the traffic flow is likened to the movement of a specific liquid, therefore, models of this class are also called hydrodynamic models. Microscopic models are those that explicitly model the movement of each car. This approach makes it possible to theoretically achieve a more accurate description of the movement of vehicles on compared to an average macro description, but this approach requires more computational resources in practical applications. Examples of such models are follow-the-leader models (see [13]), optimal-speed models (see [14]), Treiber model (see [15]), as well as models of cellular automata (see [16], [17]). Note that macroscopic models are usually used when describing the movement of road transport.

This article presents a macroscopic dynamic model describing the movement of railway transport between two node stations. The main difference from similar models (see [18], [19], [20], [21], [22]) is the rejection of the assumption that there are special roads between stations for temporary storage of part of the cargo in case of high load of stations, as well as modification of the station capacity setting. To the initial node station cargo it is received with some intensity within its capacity. Further movement of goods is carried out with intensity the value of which depends on both the capacity of the stations and the intensity of the supply of goods to the initial node station. The main objective of this study is to match the intensity of movement of goods from station to station with the intensity of supply cargo to the initial node station, which allows you to determine the practically realizable modes of cargo transportation. 


\section{Model description}

Consider the movement of goods on a railway line between two node stations connected by a set of intermediate stations. Let the number of intermediate stations be equal to $m$. Denoting by 0 and $m+1$, respectively the numbers of the initial and final node stations, we obtain the following set of station numbers: $\{0,1, . ., m, m+1\}$. We assume that the number of roads at all stations is the same and equal to $\Delta$. Each station at an arbitrary time is characterized by the number of involved roads. Denote by $z_{i}(t), i=0,1, \ldots, m+1$ the number of roads involved at $i$ - th station at time $t$. Obviously, the functions $z_{i}(t)$ must satisfy the following restrictions:

$$
0 \leq z_{i}(t) \leq \Delta, \quad i=0,1, \ldots, m, m+1
$$

Stations have a certain capacity. In this model, it is expressed as the maximum amount of cargo transported per unit of time, depending on the station load, and is described using a continuous decreasing function $\varphi($.$) , defined on$ the segment $[0, \Delta]$ and satisfying the condition $\varphi(\Delta)=0$. In this regard, each station with the number $i=1, \ldots, m$ receives cargo from the $i$ - 1 -th station with the intensity $\lambda \varphi\left(z_{i}\right)$ if $i-1$-th station is not empty and sends to the next station with the intensity $\lambda \varphi\left(z_{i+1}\right)$, if the station with the number $i$ is not empty $(0<\lambda \leq 1)$. The cargo arrives at the initial station with a certain constant intensity $d$, not exceeding $\varphi\left(z_{0}\right)$. From the final station, the cargo is distributed with an intensity of $d$ if it is not empty.

Thus, the intensity of cargo traffic at stations is set by the following system of differential equations:

$$
\begin{aligned}
\dot{z}_{0}(t)= & \min \left(d, \varphi\left(z_{0}\right)\right)-\lambda \varphi\left(z_{1}\right) \operatorname{sign}\left(z_{0}\right), \quad t \in[0,+\infty), \\
\dot{z}_{i}(t)= & \lambda \varphi\left(z_{i}\right) \operatorname{sign}\left(z_{i-1}\right)-\lambda \varphi\left(z_{i+1}\right) \operatorname{sign}\left(z_{i}\right), \\
& i=1,2 \ldots, m, \quad t \in[0,+\infty), \\
\dot{z}_{m+1}(t)= & \lambda \varphi\left(z_{m+1}\right) \operatorname{sign}\left(z_{m}\right)-d \operatorname{sign}\left(z_{m+1}\right), \quad t \in[0,+\infty) .
\end{aligned}
$$

The function $\operatorname{sign}($.$) , involved in the equations (2)-(4)$, is defined as follows:

$$
\operatorname{sign}(t)= \begin{cases}1, & \text { if } t>0 \\ 0, & \text { if } t \leq 0\end{cases}
$$

Note that the construction of the right parts of the system (2) - (4) guarantees that any solution that satisfies the condition (1) at the initial time will satisfy it at subsequent times. Everywhere in the future, we will assume that the initial condition for the system (2) - (4) satisfies (1). 
Analytical investigation of solutions of the system (2) - (4) is extremely difficult, since the right parts of differential equations are generally discontinuous functions. In this regard, the system (2) - (4) was investigated numerically. The main task of the numerical implementation of the system (2) - (4) is to study the dynamics and nature of its solutions, as well as the intensity of cargo traffic arising in the cargo transportation system, depending on the parameters of the model and the initial condition.

\section{Results of numerical experiments}

Let us proceed to presenting the results of numerical experiments, in which the function $\varphi($.$) was defined as follows:$

$$
\varphi\left(z_{i}\right)=a\left(\Delta-z_{i}\right), \quad i=0,1, \ldots, m, m+1 .
$$

Recall that the function $\varphi($.$) must be decreasing and satisfy the condition$ $\varphi(\Delta)=0$. The parameter $a$, involved in the definition of this function describes the efficiency of using roads at stations. Let us rewrite the system $(2)-(4)$, in which the function $\varphi($.$) defined according to (5),$

$$
\begin{aligned}
\dot{z}_{0}(t)= & \min \left(d, a\left(\Delta-z_{0}\right)\right) \\
& -\lambda a\left(\Delta-z_{1}\right) \operatorname{sign}\left(z_{0}\right), \quad t \in[0,+\infty), \\
\dot{z}_{i}(t)= & \lambda a\left[\left(\Delta-z_{i}\right) \operatorname{sign}\left(z_{i-1}\right)-\left(\Delta-z_{i+1}\right) \operatorname{sign}\left(z_{i}\right)\right], \\
& i=1,2 \ldots, m, \quad t \in[0,+\infty), \\
\dot{z}_{m+1}(t)= & \lambda a\left(\Delta-z_{m+1}\right) \operatorname{sign}\left(z_{m}\right) \\
& -d \operatorname{sign}\left(z_{m+1}\right), \quad t \in[0,+\infty) .
\end{aligned}
$$

From condition (1) and the definition of the $\varphi($.$) It follows that the max-$ imum flow that the stations can receive is $a \Delta$. Therefore, the parameter $d$ in the equation (6) can be represented as follows: $d=\mu a \Delta$, where $0<\mu \leq 1$. Then the system (6) - (8) takes the following form

$$
\begin{aligned}
\dot{z}_{0}(t)= & \min \left(\mu a \Delta, a\left(\Delta-z_{0}\right)\right) \\
& -\lambda a\left(\Delta-z_{1}\right) \operatorname{sign}\left(z_{0}\right), \quad t \in[0,+\infty), \\
\dot{z}_{i}(t)= & \lambda a\left[\left(\Delta-z_{i}\right) \operatorname{sign}\left(z_{i-1}\right)-\left(\Delta-z_{i+1}\right) \operatorname{sign}\left(z_{i}\right)\right], \\
& i=1,2 \ldots, m, \quad t \in[0,+\infty), \\
\dot{z}_{m+1}(t)= & \lambda a\left(\Delta-z_{m+1}\right) \operatorname{sign}\left(z_{m}\right) \\
& -\mu a \Delta \operatorname{sign}\left(z_{m+1}\right), \quad t \in[0,+\infty) .
\end{aligned}
$$


Let us move on to studying solutions to the system $(9)-(11)$. We start this study with the case when $\lambda=1$.

Investigation of solutions of the system $(9)-(11)$ for $\lambda=1$

It is easy to check that for all $0<\mu<1$ the system (9) - (11) has a stationary solution

$$
z_{i}(.) \equiv \Delta(1-\mu), i=0,1, \ldots, m+1
$$

Stationary is also any solution of the form

$$
z_{0}(.) \equiv c_{0}, 0 \leq c_{0}<\Delta(1-\mu), z_{i}(.) \equiv \Delta(1-\mu), i=1, \ldots, m+1 .
$$

Numerical experiments have shown that the stationary solution (12) is asymptotically stable in the domain $D \in R^{m+2}$, where

$$
D=\left\{x \in R^{m+2} \mid \Delta(1-\mu) \leq x_{i} \leq \Delta, i=0,1, \ldots, m+1\right\},
$$

i.e. for any solution $\left\{\tilde{z}_{i}(.)\right\}_{i=0}^{m+1}$, satisfying the condition

$$
\Delta(1-\mu) \leq \tilde{z}_{i}(0) \leq \Delta, i=0,1, \ldots, m+1
$$

will be fair

$$
\lim \left(z_{i}(t)\right)=\Delta(1-\mu) \text { for } t \rightarrow+\infty
$$

for all $i=0,1, \ldots, m+1$ (Fig. 1).

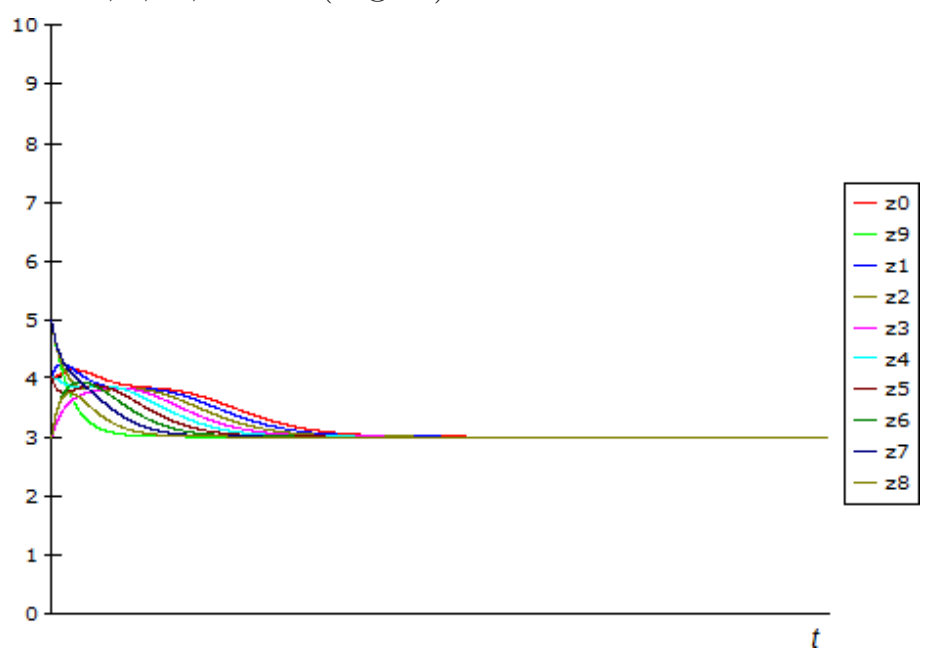


Fig 1 . The first type of solutions of system (9) - (11)

$$
(\Delta=10, a=2, \mu=0.7)
$$

At the same time, a stationary flow is established in the cargo transportation system, the value of which is equal to $\mu a \Delta$ (Fig. 2).

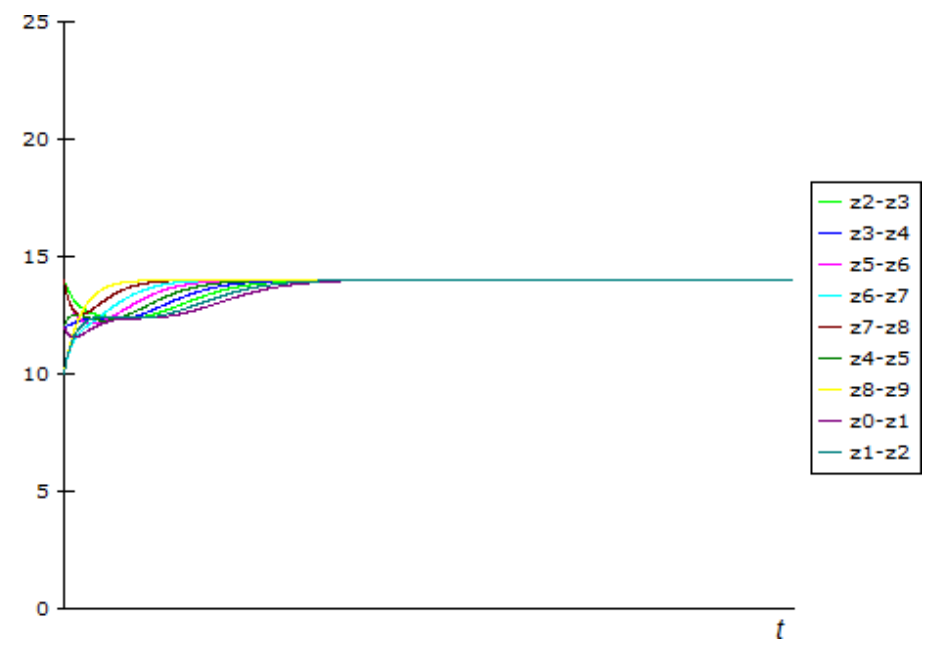

Fig. 2. Establishing a stationary flow $(\Delta=10, a=2, \mu=0.7)$

This property of solutions is also valid for $\mu=1$, although in this case the system (9) - (11) does not have a stationary solution. In this case, the stations will receive and send cargo with the maximum possible intensity equal to $a \Delta$.

Note that the satisfaction of the condition (14) is a sufficient condition for the convergence of solutions of the system (9) - (11) to the stationary solution (13), but it is not necessary.

Let us turn to the study of the properties of solutions of the system (9)-(11) in the case if the condition (14) is not satisfied. Let's start with some particular case of non-fulfillment of this condition. For this, we introduce the following notation:

$$
c=\frac{1}{m+2} \sum_{i=0}^{m+1} z_{i}(0), \quad \bar{c}=\Delta(1-\mu) \frac{m+1}{m+2} .
$$

As shown by numerical experiments, if the initial values of the system (9) - (11) do not satisfy the condition (14), but in this case $c \geq \bar{c}$, then the solution of the system (9)-(11) either converges to the stationary solution (12) (if $c \geq \Delta(1-\mu)$ ) or to one of the stationary solutions of the form (13) (if $c<\Delta(1-\mu)$ ), where 


$$
c_{0}=\Delta(1-\mu)+(c-\Delta(1-\mu))(m+1)(\text { Fig. 3). }
$$

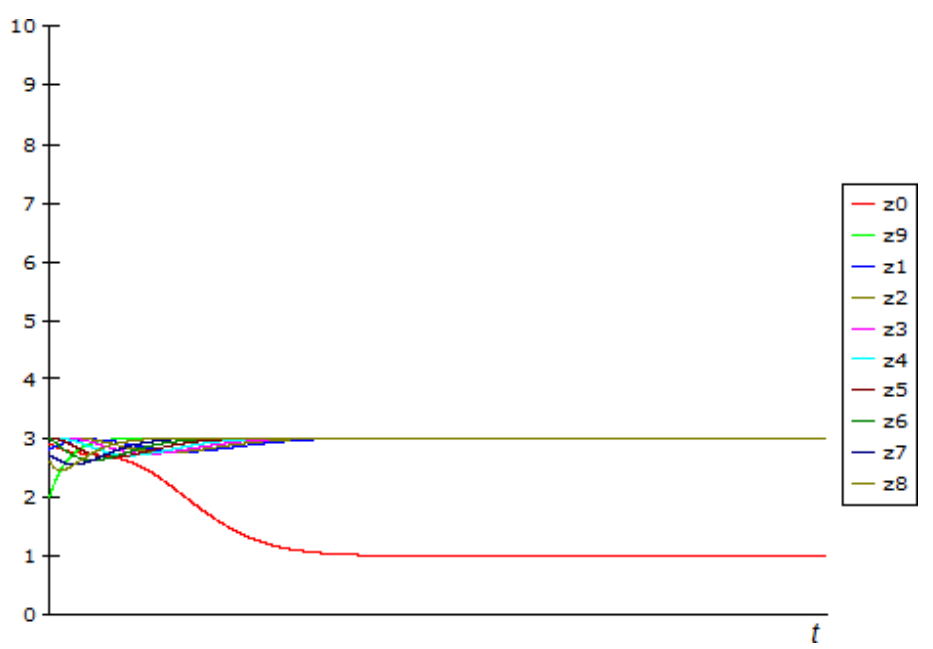

Fig 3. The second type of solution of system (9) - (11)

$$
(\Delta=10, a=2, \mu=0.7)
$$

In this case, a stationary flow is also established in the cargo transportation system, the value of which is $\mu a \Delta$.

Note that $c_{0}$ is increasing by $c$ and $c_{0}(\bar{c})=0$. For $c<\bar{c}$ the right parts of several equations of the system (9) - (11) have discontinuities, which leads to discontinuities of the corresponding components of the solution. Fig. 4 shows a graph of this solution. As you can see, starting from a certain point in time, the zero component of the solution $\left(z_{0}().\right)$ begins to fluctuate in the vicinity of zero, taking periodically zero values (suffers a break). Discontinuity of $z_{0}($. leads to discontinuity $z_{1}($.$) . This follows directly from the right side of the$ system (9) - (11). In this case, the intensity of sending goods from the zero station to the first one changes abruptly, and the magnitude of the jumps can reach a value exceeding $\mu a \Delta$ (Fig.5). 


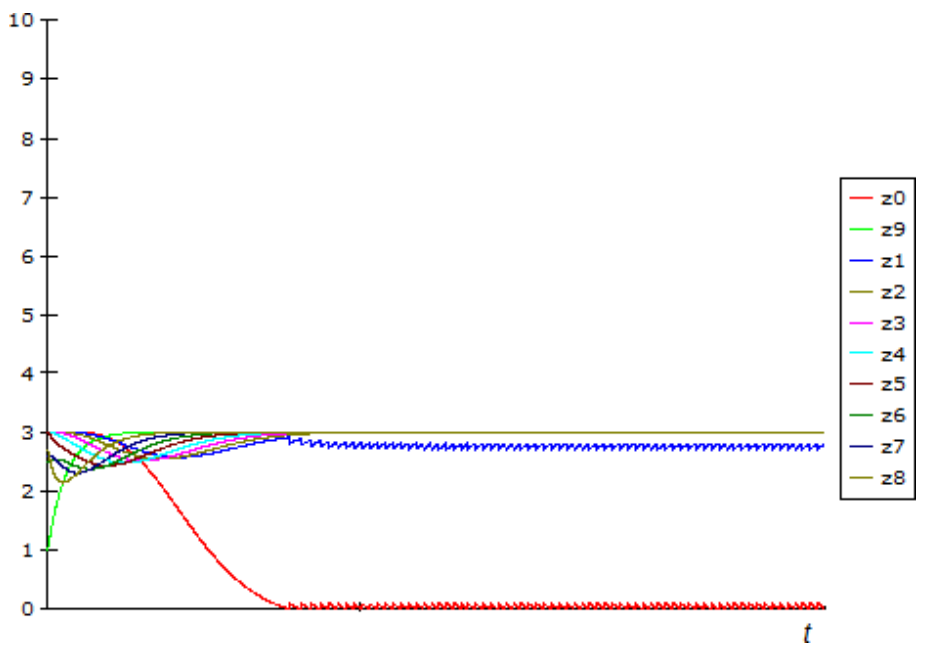

Fig 4. Discontinuous solutions of the system (9) - (11)

$$
(\Delta=10, a=2, \mu=0.7)
$$

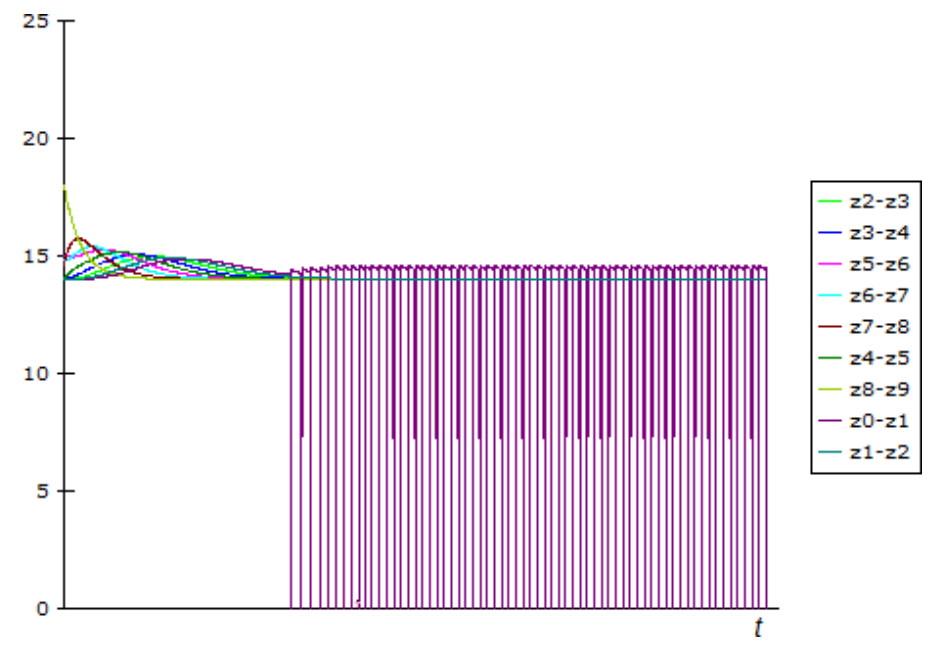

Fig 5. Abrupt flow change $(\Delta=10, a=2, \mu=0.7)$

Further reduction of the initial values (the value of $c$ ) leads to the fact that the subsequent components of the solution alternately, starting from $z_{1}($. begin to behave like $z_{0}($.$) (fluctuate in the vicinity of zero) and this leads to$ the appearance of new discontinuous components of the solution.

Let us proceed to the investigation of the general case of non-fulfillment of the condition (14). Numerical experiments have shown that the above results 
are generalized to the general case as follows: for any parameter $0<\mu<1$ there exist $0<\overline{c_{1}}<\overline{c_{2}}$ such that:

- for $c \geq \overline{c_{2}}$ the solution of the system $(9)-(11)$ is continuous and converges to the stationary solution (12) (Fig. 1) and the stationary flow is established in the cargo transportation system, the value of which $\mu a \Delta$ (the first type of solution);

- for $\overline{c_{1}}<c<\overline{c_{2}}$ the solution of the system (9) - (11) is continuous and converges to one of the stationary solutions of the form (13) (Fig. 3) and a stationary flow is established in the cargo transportation system, the value of which is also equal to $\mu a \Delta$ (the second type of solution);

- for $c \leq \overline{c_{1}}$ the solution of the system $(9)-(11)$ is discontinuous (Fig. 4), and the flow intensity between some stations changes abruptly (the third type of solution).

Thus, for $\lambda=1$ and $0<\mu<1$, depending on the initial conditions, the system $(9)-(11)$ has three types of solutions. Once again, we note that for $\mu=1$ every solution of the system (9) - (11) asymptotically converges to zero, and a stationary flow is established in the cargo transportation system, the value of which is $a \Delta$.

\section{Investigation of solutions of the system (9) $-(11)$ for $0<\lambda<1$}

Let us proceed to the study of the system (9) - (11) in the case when $0<\lambda<1$. Consider two cases: $\lambda>\mu$ and $\lambda \leq \mu$.

We start with the case $\lambda>\mu$. It is easy to check that for all $0<\mu<1$ and $\mu<\lambda<1$ the system (9) - (11) has a stationary solution

$$
z_{0}(.) \equiv \Delta(1-\mu), z_{i}(.) \equiv \Delta\left(1-\frac{\mu}{\lambda}\right), i=1, \ldots, m+1 .
$$

Stationary is also any solution of the form

$$
z_{0}(.) \equiv \tilde{c_{0}}, 0 \leq \tilde{c_{0}}<\Delta(1-\mu), z_{i}(.) \equiv \Delta\left(1-\frac{\mu}{\lambda}\right), i=1, \ldots, m+1 .
$$

Numerical experiments have shown that the stationary solution (16) is asymptotically stable in the domain $\tilde{D} \in R^{m+2}$, where

$$
\begin{aligned}
& \tilde{D}=\left\{x \in R^{m+2} \mid \Delta(1-\mu) \leq x_{0} \leq \Delta,\right. \\
& \left.\Delta\left(1-\frac{\mu}{\lambda}\right) \leq x_{i} \leq \Delta, i=1, \ldots, m+1\right\} .
\end{aligned}
$$

Let us go to the description of the nature of the solutions of the system (9) - (11). 
It was revealed that for any initial values of the system (9) - (11) (value $c$ ) and the parameter $\mu$ there exists $\tilde{\lambda}_{1}(c, \mu), \tilde{\lambda}_{2}(c, \mu), \quad \mu<\tilde{\lambda}_{1}(c, \mu)<\tilde{\lambda}_{2}(c, \mu) \leq 1$ such that the solution of the system $(9)-(11)$ is continuous for any value of the parameter $\lambda$ from the segment $\left[\mu, \tilde{\lambda}_{2}(c, \mu)\right]$ and is discontinuous for any $\lambda$ from the half-interval $\left(\tilde{\lambda}_{2}(c, \mu), 1\right]$ if $\tilde{\lambda}_{2}(c, \mu)<1$. Moreover, for any $\lambda$ from the segment $\left[\mu, \tilde{\lambda}_{1}(c, \mu)\right]$ it converges to a stationary solution (16) (the first type of solution), and for any $\lambda$ from the half-interval $\left(\tilde{\lambda}_{1}(c, \mu), \tilde{\lambda}_{2}(c, \mu)\right]$ converges to one of the stationary solutions of the form (17) (the second type of solution). In both cases, a stationary flow is established in the cargo transportation system, the value of which is $\mu a \Delta$. Values $\tilde{\lambda}_{1}(c, \mu)$ and $\tilde{\lambda}_{2}(c, \mu)$ are decreasing in both variables. As with $\lambda=1$ discontinuous solutions will represent the third type of solution. This type of solution is characterized by the fact that the flow intensity between some stations changes abruptly.

Finally, we investigate the solution of the system (9) - (11) in the case when $\lambda \leq \mu$. It is easy to check that in this case the system (9)-(11) has a stationary solution

$$
z_{0}(.) \equiv \Delta(1-\lambda), z_{i}(.) \equiv 0, i=1, \ldots, m+1 .
$$

Numerical experiments have shown that this stationary solution is asymptotically stable on the whole set of admissible initial values of the system (9) - (11), i.e. on the set

$$
\tilde{\tilde{D}}=\left\{x \in R^{m+2} \mid 0 \leq x_{i} \leq \Delta, i=0,1, \ldots, m+1\right\} .
$$

In this case, a stationary flow is established in the cargo transportation system, the value of which is $\lambda a \Delta$.

So, one more type of solution of the system (9) - (11) (fourth) has been identified. Note that three types of solutions out of four (except for the third) combine the following properties: continuity of the solution and convergence to a certain stationary solution, leading to the establishment of a stationary flow in the system of cargo transportation. The main thing is the fact that all three of these types of solutions describe practically realizable modes of cargo transportation. Recall that discontinuous solutions (the third type of solutions) generate abrupt changes in the intensity of freight traffic. In practice, such a cargo transportation regime is extremely difficult to implement.

\section{Conclusion}

This article examines the model of organization of railway cargo transportation on the section of the railway network between two node stations. The dynamics 
of flow depending on the intensity of cargo supply to the initial node station and the rules of cargo movement from one station to another was investigated. The central point in this rule is the use of station capacity, which is set as the maximum the possible intensity of cargo reception by the station per unit of time, depending on its load. This model is set by the system of differential equations with discontinuous right parts, which has been studied numerically. As a result of numerical experiments were possible types of solutions of this system are identified, most of which determine the practically realizable modes of cargo transportation. Such modes of cargo transportation determine the dynamics of the flow on a given section of the railway network.

\section{References}

[1] V.I. Shvetsov, Mathematical modeling of traffic flows, Automation and Remote Control, 64, No 11 (2003), 1651-1689.

[2] A.G. Wilson, A family of spatial interaction models and associated developments, Environment and Planning A, 3, No 1 (1971), 1-32.

[3] B. Harris, A.G. Wilson, Equilibrium values and dynamics of attractiveness terms in production-constrained spatial-interaction models, Environment and Planning A, 10, No 4 (1978), 371-388.

[4] A.S. Fotheringham, A new set of spacial-interaction models: the theory of competing destinations, Environment and Planning A, 15, No 1 (1983), 15-36.

[5] A.S. Fotheringham, Modelling hierarchical destination choice, Environment and Planning A, 18, No 3 (1986), 401-418.

[6] V.I. Shvetsov, Algorithms for distributing traffic flows, Automation and Remote Control, 70, No 10 (2009), 1728-1736.

[7] T. Leventhal, G.L. Nemhauser, L. Trotter, A column generation algorithm for optimal traffic assignment, Transportation Science, 7, No 2 (1973), 168-176.

[8] H.K. Lo, A. Chen, Traffic equlibrium problem with route-specific costs: formulation and algorithms, Transportation Research: Part B, 34, No 6 (2000), 493-513. 
[9] H. Bar-Gera, Origin-based algorithm for the traffic assignment problem, Transportation Science, 36, No 4 (2002), 398-417.

[10] C.F. Daganzo, The cell transmission model: A dynamic representation of highway traffic consistent with the hydrodynamic theory, Transportation Research B, 28, No 4 (1994), 269-287.

[11] C.F. Daganzo, The cell transmission model, Part II: Network traffic, Transportation Research B, 29, No 2 (1995), 79-93.

[12] H. Inose, T. Hamada, Road Traffic Control, University of Tokyo Press, Tokyo (1975).

[13] M. Brackstone, M. McDonald, Car following: A historical review, Transportation Research F, 2, No 4 (1999), 181-196.

[14] M. Bando, K. Hasebe, A. Nakayama, A. Shibata, Y. Sugiyama, Dynamical model of traffic congestion and numerical simulation, Physical Review E, 51, No 2 (1995), 1035-1042.

[15] M. Treiber, A. Hennecke, D. Helbing, Congested traffic states in empirical observations and microscopic simulations, Physical Review E, 62, No 2 (2000), 1805-1824.

[16] M. Cremer, J. Ludwig, A fast simulation model for traffic flow on the basis of Boolean operations, Mathematics and Computers in Simulation, 28, No 4 (1986), 297-303.

[17] D. Chowdhury, L. Santen, A. Schadschneider, Statistical physics of vehicular traffic and some related systems, Physics Reports, 329, No 4-6 (2000), 199-329.

[18] L.A. Beklaryan, N.K. Khachatryan, Traveling wave type solutions in dynamic transport models, Functional Differential Equations, 13, No 12 (2006), 125-155.

[19] L.A. Beklaryan, N.K. Khachatryan, On one class of dynamic transportation models, Computational Mathematics and Mathematical Physics, 53, No 10 (2013), 1466-1482.

[20] N.K. Khachatryan, A.S. Akopov, Model for organizing cargo transportation with an initial station of departure and a final station of cargo distribution, Business Informatics, 39 (2017), 25-35. 
[21] N.K. Khachatryan, A.S. Akopov, F.A. Belousov, About quasi-solutions of traveling wave type in models for organizing cargo transportation, Business Informatics, 43 (2018), 61-70.

[22] L.A. Beklaryan, N.K. Khachatryan, A.S. Akopov, Model for organization cargo transportation at resource restrictions, International Journal of Applied Mathematics, 32, No 4 (2019), 627-640; DOI: 10.12732/ijam.v32i4.7. 
